\title{
Psychological health problems among Syrians during war and the COVID-19 pandemic: national survey
}

\author{
Fatema Mohsen, ${ }^{1} \odot$ Batoul Bakkar, ${ }^{2}$ Sara Melhem, ${ }^{3}$ Samar Aldakkak, ${ }^{4}$ \\ Dana Mchantaf, ${ }^{5}$ Marah Marrawi ${ }^{6}$ and Youssef Latifeh $^{7}$
}

\begin{abstract}
${ }^{1}$ Faculty of Medicine, Syrian Private University, Damascus, Syria. Email: fatemamohsena@ fatemamohsena@ gmail.com

${ }^{2}$ Faculty of Medicine, Syrian Private University, Damascus, Syria

${ }^{3}$ Faculty of Medicine, Syrian Private University, Damascus,

Syria

${ }^{4}$ Faculty of Medicine, Syrian Private University, Damascus,

${ }^{5}$ Faculty of Medicine, Syrian Private University, Damascus, Syria

${ }^{6}$ Department of Statistics, Syrian Private University, Damascus, Syria

${ }^{7}$ Department of Psychiatry, Faculty of Medicine, Syrian Private University, Damascus, Syria; and Department of Psychiatry, Faculty of Medicine, Damascus University, Damascus, Syria
\end{abstract}

Keywords. Anxiety disorders; depressive disorders; low and (a) national survey; war and pandemic.

First received 27 Dec 2020 Final revision 19 Feb 2021 Accepted 8 Mar 2021

doi:10.1192/bji.2021.16

(c) The Author(s), 2021. Published by Cambridge University Press on behalf of the Royal College of Psychiatrists. This is an Open Access article, distributed under the terms of the Creative Commons Attribution licence (http://creativecommons.org/ licenses/by/4.0/), which permits unsticts bution, and reproduction in any medium provided the original work is properly cited.
This study aims to assess the prevalence of depression and anxiety during the COVID-19 outbreak embedded within the war in Syria. A web-based cross-sectional survey design was employed. The 9-item Patient Health Questionnaire and the 7-item Generalized Anxiety Disorder scale revealed a high prevalence of depressive $(n=3326 ; 83.4 \%)$ and anxiety symptoms $(n=2777 ; 69.6 \%)$ among the 3989 participants. Multivariable logistic regression analysis was performed to identify factors associated with depression and anxiety. There is an urgent need within the healthcare system in Syria to provide mental healthcare to alleviate acute mental health disturbances and associated physical health perceptions among Syrians.

The Syrian conflict, now spanning a decade, paralleled with the domestically spreading coronavirus disease 2019 (COVID-19), is threatening the lives of civilians across Syria. The mental health of Syrians has been immensely challenged during the viral pandemic that emerged in this ongoing crisis. Healthcare systems have been pushed to breaking point to deal with the pandemic, with the ever-increasing number of COVID-19-related deaths and confirmed cases across the country leading to public trepidation. This sudden outbreak of a deadly virus is bound to trigger anxiety, depression and other mental health issues among the population. Lack of effective treatment, fears of death and contagion, losing loved ones, social isolation, media misinformation overload, depletion of stocks of personal protection equipment, panic buying and inadequate psychosocial support may all adversely affect the individual's mental health. Worldwide, levels of anxiety symptoms have ranged from 6.3 to $50.9 \%$ and depressive symptoms from 17.2 to $53.5 \%$ during the COVID-19 pandemic. $^{1}$

The prolonged conflict in Syria has exposed civilians to grave human rights violations, including killing, maiming, sexual assault, torture, and displacement, where $89 \%$ of the population are living in extreme poverty. ${ }^{2}$ Infrastructures, including hospitals, schools and public services, are being systematically decimated. For instance, the Syrian Ministry of Health provides treatment for mental illness and substance misuse in three hospitals: Ibn Sina Hospital (rural Damascus),
Ibn Rushd Hospital (Damascus) and Ibn Khaldoun Hospital (Aleppo); the last was bombarded on 25 December 2012. ${ }^{3}$ One million Syrians are estimated to suffer from severe psychiatric disorders, with only 80 psychiatrists working in Syrian territories ( 1 per 100000 population) in 2018. , $3^{2}$ Assuming that psychiatrists work 5 days/ week for 52 weeks/year, and that each doctor can follow up on 15 patients/day and that they do not follow up on each patient $>3$ times/year, the total number of patients that the 80 psychiatrists can follow up on annually is 104000 ; that leaves $90 \%$ of patients unattended, untreated and unmanaged. The combination of the above is set to overwhelm health services. ${ }^{3}$

Currently, there are no published studies on the simultaneous impact of war and pandemics; however, a recent systematic review on the mental health of Syrian refugees and Syrians revealed that depression ranged from 11 to $49 \%$ and anxiety from 49 to $55 \%{ }^{4}$ A recently published study showed alarming numbers, where $60 \%$ of the Syrian population are suffering from symptoms consistent with moderate to severe mental disorder. ${ }^{5}$ We describe here the first study that aimed to provide an estimated prevalence of depressive and anxiety symptoms among Syrians during the civil war and COVID-19 pandemic. The objective was to identify potential factors associated with depressive and anxiety symptoms.

\section{Method}

This web-based cross-sectional study was conducted in May 2020. All participants aged 18 and above, residing in Syria, who completed the survey were included in the study. The questionnaire was distributed through various social media platforms. The Arabic versions of the 9-item Patient Health Questionnaire (PHQ-9) and the 7-item Generalized Anxiety Disorder (GAD-7) scale were used to evaluate the symptoms of depression and anxiety respectively. ${ }^{6}$ The PHQ-9 and GAD-7 cut-off scores were both 0$4^{7,8}$ The study was ethically approved by the Institutional Review Board of the Faculty of Medicine, Syrian Private University. The survey is shown in supplementary Appendix 1, available at https://dx.doi.org/10.1192/bji.2021.16. All participants provided written informed consent to participate in the study. Data analysis was conducted using the Statistical Package for Social Sciences version 25.0 for Windows. The chi-squared test 
Table 1

Sociodemographic characteristics: $(n=3989)$

\begin{tabular}{|c|c|}
\hline & $n(\%)$ \\
\hline \multicolumn{2}{|l|}{ Gender } \\
\hline Male & $1054(26.4)$ \\
\hline Female & $2935(73.5)$ \\
\hline \multicolumn{2}{|l|}{ Age, years } \\
\hline $18-25$ & 2870 (71.9) \\
\hline $26-34$ & $685(17.2)$ \\
\hline $35-44$ & $261(6.5)$ \\
\hline $45-54$ & $121(3)$ \\
\hline$>55$ & $52(1.4)$ \\
\hline \multicolumn{2}{|l|}{ Social status } \\
\hline Single & $3096(77.6)$ \\
\hline Married & $714(17.9)$ \\
\hline Other & $179(4.5)$ \\
\hline \multicolumn{2}{|l|}{ Economic status } \\
\hline Excellent & $251(6.3)$ \\
\hline Good & $1800(45.1)$ \\
\hline Moderate & $1522(38.1)$ \\
\hline Poor & $416(10.4)$ \\
\hline \multicolumn{2}{|l|}{ Chronic disease(s) } \\
\hline Yes & $556(13.9)$ \\
\hline No & $3433(86)$ \\
\hline \multicolumn{2}{|l|}{ Education } \\
\hline Primary school & $21(0.5)$ \\
\hline Intermediate school & $115(2.9)$ \\
\hline Secondary school & $370(9.3)$ \\
\hline College/university & $3271(82)$ \\
\hline Master's degree & $185(4.6)$ \\
\hline $\mathrm{PhD}$ & $27(0.7)$ \\
\hline \multicolumn{2}{|l|}{ Occupation } \\
\hline Healthcare worker & $259(6.5)$ \\
\hline Government institution & $239(6)$ \\
\hline Private institution & $202(5.1)$ \\
\hline Business & $202(5.1)$ \\
\hline Military & $35(0.9)$ \\
\hline Student & $2397(60.1)$ \\
\hline Other & $655(16.4)$ \\
\hline \multicolumn{2}{|l|}{ Household members } \\
\hline Alone & $54(1.4)$ \\
\hline $1-5$ & $2474(62)$ \\
\hline$>5$ & $1461(36.6)$ \\
\hline
\end{tabular}

was applied to describe the relationship between sociodemographic variables and the severity of anxiety and depression. Binary logistic regression analysis was conducted to determine the significant predictors of depressive and anxiety symptoms using the sociodemographic variables as the independent variables.

\section{Results}

Of the 5000 total participants invited to take part in the study, the final sample was 3989 participants (a response rate of 79.8\%). Sociodemographic characteristics are displayed in Table 1.

The prevalence of depressive and anxiety symptoms among participants was 3326 (83.4\%) and $2777(69.6 \%)$ respectively. Depression severity: none, 663 (16.6\%); mild, 1150 (28.8\%); moderate, 1006 (25.2\%); moderately severe, 684 $(17.2 \%)$; and severe, $486(12.2 \%)$. Anxiety severity: none, 1212 (30.4\%); mild, 1293 (32.4\%); moderate, 805 (20.2\%); and severe $679(17.0 \%)$.

Depression scores were significantly higher among participants who were single $(P<$ $0.0001)$, had college/university level education $(P<0.0001)$, were students by occupation $(P<$ $0.0001)$, lived with 1-5 household members $(P<0.0001)$, had a history of chronic disease(s) $(P<0.0001)$ and were of good economic status $(P<0.0001)$. Anxiety scores were significantly higher among those with college/university level education $(P<0.0001)$, were students by occupation $(P<0.0001)$, lived with $1-5$ household members $(P=0.014)$, had a history of chronic disease(s) $(P<0.0001)$ and were of good economic status $(P<0.0001)$ (supplementary Appendix 2).

Multiple logistic regression analysis showed that the following factors were significantly associated with depressive and anxiety symptoms: female gender; age 18-25 years; occupation in private, business and other sectors, as well as students; poor, moderate and good economic status; and having chronic disease(s), (Table 2).

\section{Discussion}

The prevalence of depressive 3326 (83.4\%) and anxiety 2777 (69.6\%) symptoms among Syrians was significantly higher compared with the prevalence of anxiety in 17 studies $(31.9 \%)$ and the prevalence of depression in 14 studies $(33.7 \%){ }^{1}$ The high prevalence of depressive and anxiety symptoms found in our study surpasses World Health Organization prevalence estimations (of $3.9 \%$ and $4.3 \%$ respectively). ${ }^{9}$ A potential explanation for this disparity is that exposure to multiple stressors, including war and pandemic, may have triggered mental disorders among Syrians.

Literature has revealed that Syria's lack of mental healthcare services provided by psychologists and psychiatrists results in limited access to mental healthcare for many distressed individuals. Scarcity of online and social media support in the form of publications, resources, posts, guidelines, videos and online group chats is likely to exacerbate the problem., ${ }^{2,3}$ Such support can alleviate mental health problems caused by risk factors such as exposure to COVID-19.

All participants presenting with mild (1150; $28.8 \%)$ and moderate $(1006 ; 25.2 \%)$ depression and mild $(1293 ; 32.4 \%)$ and moderate (805; $20.2 \%$ ) anxiety require clinical assessment for symptom duration and functional disability to determine the necessity of treatment. As over half the population (54\% for depression and $52.6 \%$ for anxiety) requires a scheduled clinical assessment, with a total population of 18284 
Table 2

Multiple logistic regression analysis of depressive and anxiety symptoms

\begin{tabular}{|c|c|c|c|c|c|}
\hline & & \multirow[b]{2}{*}{$P$} & \multirow[b]{2}{*}{ OR } & \multicolumn{2}{|c|}{$95 \%$ CI for OR } \\
\hline & & & & Lower & Upper \\
\hline \multicolumn{6}{|l|}{ Depression } \\
\hline Gender & Female $v$. male & $<0.0001$ & 1.653 & 1.355 & 2.017 \\
\hline \multirow[t]{4}{*}{ Age } & $26-34$ v. $18-25$ & 0.015 & 0.713 & 0.544 & 0.936 \\
\hline & $35-44$ v. $18-25$ & $<0.0001$ & 0.430 & 0.297 & 0.622 \\
\hline & $45-54$ v. $18-25$ & $<0.0001$ & 0.298 & 0.184 & 0.482 \\
\hline & 55 and above $v .18-25$ & $<0.0001$ & 0.156 & 0.080 & 0.308 \\
\hline \multirow[t]{4}{*}{ Occupation } & Private $v$. healthcare worker & 0.010 & 1.825 & 1.152 & 2.891 \\
\hline & Business $v$. healthcare worker & 0.003 & 2.058 & 1.280 & 3.310 \\
\hline & Student $v$. healthcare worker & $<0.0001$ & 2.315 & 1.658 & 3.234 \\
\hline & Other $v$. healthcare worker & 0.001 & 1.809 & 1.259 & 2.599 \\
\hline \multirow[t]{3}{*}{ Economic status } & Poor $v$. excellent & $<0.0001$ & 4.771 & 2.956 & 7.698 \\
\hline & Moderate $v$. excellent & $<0.0001$ & 2.075 & 1.473 & 2.922 \\
\hline & Good $v$. excellent & 0.011 & 1.531 & 1.101 & 2.130 \\
\hline Chronic disease(s) & Yes $v$. no & $<0.0001$ & 2.990 & 2.120 & 4.215 \\
\hline \multicolumn{6}{|l|}{ Anxiety } \\
\hline Gender & Female $v$. male & $<0.0001$ & 1.476 & 1.263 & 1.725 \\
\hline \multirow[t]{4}{*}{ Age } & $26-34$ v. $18-25$ & 0.001 & 0.708 & 0.577 & 0.869 \\
\hline & $35-44$ v. $18-25$ & $<0.0001$ & 0.328 & 0.234 & 0.459 \\
\hline & $45-54$ v. $18-25$ & $<0.0001$ & 0.243 & 0.154 & 0.384 \\
\hline & 55 and above $v$. $18-25$ & $<0.0001$ & 0.189 & 0.100 & 0.357 \\
\hline Education & University/college, Master's, PHD v. primary, intermediate, secondary & $<0.0001$ & 0.624 & 0.488 & 0.798 \\
\hline Occupation & Healthcare worker $v$. government, private, business, military, student, other & 0.021 & 0.725 & 0.552 & 0.953 \\
\hline \multirow[t]{3}{*}{ Economic status } & Poor v. excellent & $<0.0001$ & 6.339 & 4.390 & 9.153 \\
\hline & Moderate $v$. excellent & $<0.0001$ & 3.140 & 2.371 & 4.160 \\
\hline & Good $v$. excellent & 0.008 & 1.451 & 1.101 & 1.911 \\
\hline Chronic disease(s) & Yes v. no & $<0.0001$ & 2.492 & 1.941 & 3.198 \\
\hline
\end{tabular}

423, this means that over 9 million people require mental health services. The lack of psychiatrists, hospitals, medical capacity and clinics projects ominous outcomes for Syrians' mental health.

Participants with moderately severe (684; $17.2 \%)$ and severe $(486 ; 12.2 \%)$ depression and severe $(679 ; 17.0 \%)$ anxiety may require further assessment and active treatment with psychotherapy and/or medications. In 2005, the health expenditure per capita in Syria was US\$58, which is extremely low in comparison with the UK (US\$2900). ${ }^{10}$ As the country is drowning in debts from the effects of the rampant war, the health expenditure per capita is likely to be significantly lower in the coming years. In 2013, the World Federation for Mental Health (WFMH) declared a call for action on the mental health consequences of the manifold emergency in Syria. One of WFMH's concerns is the neglect of trauma created by the violence and disruption.

Multivariable logistic regression analyses found strong associations with depressive and anxiety symptoms for: female gender; the 18-25 age group; careers in private, business and 'other' sectors, as well as students; poor, moderate and good economic status; and having chronic disease(s). This last may be attributed to the higher incidence of depression and anxiety among chronically ill people than others. ${ }^{11}$ The COVID-19 pandemic is likely to add to the problem, given that people with chronic disease(s) are at increased risk of COVID-19 infection, which can be more severe, with increased rates of complications and mortality. ${ }^{12}$ The findings regarding young adults may be related to the fact that this age group, who were children when this war started, have grown up witnessing destruction and displacement and grave violations of children's rights - abductions, rape, killing and maiming continue unabated. Approximately 6 million Syrian children have been born since the crisis began, growing up knowing nothing but war, terror and displacement. ${ }^{13}$ Prolonged lockdowns that have harmed society, left many people unemployed, schools and universities closed and resulted in hyperinflation could explain the spread of mental disorders among younger generations. The findings related to females reflect the literature, which reports an increased prevalence of depression in women associated in part with female sex hormones and with factors such as life circumstances and culture. ${ }^{14}$ Also, this 
conflict has rendered Syrian women and girls particularly vulnerable to physical, sexual, economic and psychological violence. ${ }^{2}$

For the estimated high burden of depressive and anxiety symptoms encountered in this study, we must adopt the epidemiological modelling outputs for targeted mental health planning to calculate the required resources needed to guide optimal mental health service delivery. ${ }^{15}$

\section{Limitations}

Our findings may not generalise to the general population, as well-educated Syrians of good socioeconomic status are over-represented in the sample. Elderly Syrians who were underrepresented in this study, which may be due to limited access to the internet are more likely to exhibit symptoms associated with depression and anxiety, as the elderly have a higher prevalence of chronic diseases. ${ }^{11}$ Self-reporting has certain limitations compared with structured interviews. The crosssectional design of the study does not allow inferences to be drawn about causality.

\section{Supplementary material}

Supplementary material is available online at https://doi.org/10. 1192/bji.2021.16

\section{Data availability}

All data related to this paper's conclusions are available and stored by the authors. All data are available from the corresponding author on reasonable request.

\section{Acknowledgements}

We are thankful to the management of the Syrian Private University for support in the field of medical training and research. We thank everyone who participated in this study.

\section{Author contributions}

F.M. and B.B. conceptualised the study, participated in the design, wrote the study protocol, performed the statistical analysis, did a literature search and drafted the manuscript. S.M. participated in statistical analysis. D.M., S.A. and Y.L. did a literature search and revision of the draft. All authors read and approved the final draft.

\section{Funding}

This research received no specific grant from any funding agency, commercial or not-for-profit sectors.

\section{Declaration of interest}

None.

\section{References}

1 Salari N, Hosseinian-Far A, Jalali R, Vaisi-Raygani A, Rasoulpoor S, Mohammadi M, et al Prevalence of stress, anxiety, depression among the general population during the COVID-19 pandemic: a systematic review and meta-analysis. Global Health 2020; 16 (1): 57

2 Hedar M. Mental health during the Syrian crisis: how Syrians are dealing with the psychological effects. Int Rev Red Cross 2017; 99: 927

3 Abou-Saleh M, Mobayed M. Mental health in Syria. Int Psychiatry 2013; 10: 58-60

4 Hendrickx M, Woodward A, Fuhr DC, Sondorp E, Roberts B. The burden of mental disorders and access to mental health and psychosocial support services in Syria and among Syrian refugees in neighboring countries: a systematic review. J Public Health 2020; 42: e299-e310.

5 Kakaje A, Al Zohbi R, Aldeen OH, Makki L, Alyousbashi A, Alhaffar MBA. Mental disorder and PTSD in Syria during wartime: a nationwide crisis. BMC Psychiatry 2021; 21(1): 2

6 Sawaya H, Atoui M, Hamadeh A, Zeinoun P, Nahas Z. Adaptation and initial validation of the Patient Health Questionnaire - 9 (PHQ-9) and the Generalized Anxiety Disorder 7 Questionnaire (GAD-7) in an Arabic speaking Lebanese psychiatric outpatient sample. Psychiatry Res 2016; 239: 245-52.

7 Kocalevent R-D, Hinz A, Brähler E. Standardization of the depression screener patient health questionnaire (PHQ-9) in the general population. Gen Hosp Psychiatry 2013; 35: 551-5.

8 Löwe B, Decker O, Müller S, Brähler E, Schellberg D, Herzog W, et al Validation and standardization of the Generalized Anxiety Disorder Screener (GAD-7) in the general population. Med Care 2008; 46: 266-74

9 World Health Organization. Depression and Other Common Mental Disorders: Global Health Estimates. World Health Organization, 2017.

10 Assalman I, Alkhalil M, Curtice M. Mental health in the Syrian Arab Republic. Int Psychiatry 2008; 5: 64-6.

11 Gerontoukou EI, Michaelidoy S, Rekleiti M, Saridi M, Souliotis K. Investigation of anxiety and depression in patients with chronic diseases. Health Psychol Res 2015; 3(2): 2123.

12 Zhou F, Yu T, Du R, Fan G, Liu Y, Liu Z, et al Clinical course and risk factors for mortality of adult inpatients with COVID-19 in Wuhan, China: a retrospective cohort study. Lancet 2020; 395: 1054-62.

13 UNICEF. Syrian Crisis: After a Decade of Conflict, Children Continue to Pay the Heaviest Price. UNICEF, 2021 (https://www. unicef.org/emergencies/syrian-crisis).

14 Albert PR. Why is depression more prevalent in women?. Psychiatry Neurosci 2015; 40: 219-21

15 Charlson F, Lee Y, Diminic S, Whiteford H. Applications of the epidemiological modelling outputs for targeted mental health planning in conflict-affected populations: the Syria case-study. Glob Ment Health 2016: 3: e8. 